\title{
Teach Your Children Well: Stage IV Colorectal Cancer and Variability in Practice Patterns
}

\author{
William R. Jarnagin, $\mathrm{MD}^{1}$, William C. Chapman, $\mathrm{MD}^{2}$, and Sharon Weber, $\mathrm{MD}^{3}$ \\ ${ }^{1}$ Department of Surgery, MSKCC, New York, NY; ${ }^{2}$ Section of Transplantation, Washington University, St. Louis, MO; \\ ${ }^{3}$ University of Wisconsin Hospitals, Madison, WI
}

Training surgeons, like raising children, involves a certain element of uncertainty-the mentor, or the parent, provides the ingredients necessary for success, and the progeny are then released into the world, fingers crossed. Generally, things work out as planned, and while there are many different pathways to success, the important lessons and the core values do not waiver-like surgical training programs, no two families are the same but the fundamentals do not vary.

The recipe for training surgeons, like parenting, has been essentially a trial-and-error process, evolving over time, during which the essential elements required have been forged. Whereas training programs have changed and adapted in response to changes in surgical practice, the basic principles, or core values, remain. The clinical problems and operative approaches may change, but the clinical judgment and reasoning skills required for mature surgical decision-making do not. Clearly, no amount of training can provide exposure to every possible situation that one might encounter, nor can it account for changes in practice that normally occur over time. Like a parent raising a child the most that a training program can hope to accomplish is to provide the necessary foundation to adapt appropriately to a changing world.

In the current issue of Annals of Surgical Oncology, Nathan et al. ${ }^{1}$ analyze practice patterns among surgeons treating patients with stage IV colorectal cancer. In this interesting and well-conducted investigation, the authors presented practicing surgeons, reportedly with an "interest" in liver surgery, with several clinical scenarios and analyzed the responses. The study population of 219 , which

(C) Society of Surgical Oncology 2012

Published Online: 1 September 2012

W. R. Jarnagin, MD

e-mail: jarnagiw@mskcc.org was derived from an initial email invitation list of 1,032, was largely from academic centers (79\%), fellowship trained (51\% surgical oncology, $25 \% \mathrm{HPB}$, and $21 \%$ transplant), clinically oriented (75\% time in clinical care), and reasonably experienced (median of 12 years in practice and 30 self-reported liver resections per year for all indications).

In general, responses appeared to be within the main stream of attitudes, with the overwhelming majority agreeing that hepatic resection is potentially curative therapy (99 \%) but a much smaller proportion (67\%) conferring the same degree of efficacy to ablative therapy. Appropriately, the disease extent did have some impact on the respondents' choice of therapy: liver resection, chemotherapy followed by liver resection ("neoadjuvant"), ablation, or palliative chemotherapy. Although there was no consensus regarding the use of neoadjuvant chemotherapy versus initial surgery, when the former was recommended, the respondent's displayed a good understanding of its role, with the overwhelming majority recommending a limited course and not treatment to maximal response.

With more in-depth analysis, greater variability became apparent. The authors noted that different clinical characteristics influenced treatment recommendations to varying degrees, with timing of metastatic disease presentation, tumor number and location, as well as the presence of extrahepatic disease having the largest impact. In general, there was a greater preference for initial chemotherapy in patients with synchronous liver metastases and more advanced disease, whereas there was a strong aversion to recommending hepatic resection in patients with extrahepatic disease at any site.

A major finding of the study was the identification of physician-related variables that impacted treatment recommendations. Specifically, the authors found that surgical oncology-trained surgeons were more likely to recommend 
neoadjuvant or palliative chemotherapy versus initial resection in clinical settings associated with more advanced disease compared with their HPB/transplant colleagues. There also was a similar reluctance to consider resection among surgeons with greater years in practice.

The results of this study might initially be unsettling to some degree. After all, should we not expect more uniformity or consensus regarding treatment recommendations? However, on further consideration, the results should not come as a great surprise. First and foremost, just as a family shapes a child's view of the world, training programs exert great influence over a surgeon's approach to clinical problems, and we would expect this to be particularly so when the level of evidence supporting one approach over another is low. This was recently affirmed in a report by In et al., which evaluated practice patterns in NCI-designated cancer centers compared with other institutions. There was little variation observed when guidelines were supported by level 1 evidence; however, variation in care was greater when guidelines were based on expert consensus rather than data from prospective clinical trials. ${ }^{2}$

Many of the issues pertaining to management of stage IV colorectal cancer addressed in the present study, unfortunately, fall into the latter category. For several of the clinical scenarios presented, appropriate management remains the subject of ongoing debate, and although experts in the field might agree generally on the management strategy, significant variability in treatment recommendations would be expected. In these situations, where black and white gives way to many shades of gray, is it not expected that a surgeon's approach will reflect that of his/her mentors or professional family? That is not to say that one should blindly accept all of these lessons in perpetuity. On the contrary, if a training program has done its job, a healthy dose of skepticism and questioning of "dogma" should be expected, and indeed, often is the best way forward. This may, in part, explain the differences in treatment recommendations among older surgeons observed in the present study.

Overall, the respondents seemed to mirror the uncertainty in the field to a large degree; they appeared to get the important questions correct but showed variability in their responses when there was a dearth of data supporting one approach over another. The results should not be taken as an indictment of surgeons for failing to understand the correct treatment nor of surgical training programs for failing to provide proper guidance. On the contrary, the data seem to paint a picture of surgeons relying on their training (or professional "family values") as they negotiate a changing, and at times confusing, clinical landscape.

Before sweeping generalizations can be made regarding the influence of training on practice patterns, certain limitations of this study must be acknowledged. First, an element of bias would seem inevitable given the low response rate, which was just more than $20 \%$ of all invitations sent. Second, combining HPB and transplant fellowship-trained surgeons into one group, although reasonable in order to balance the numbers, is suboptimal given inherent differences in training focus. Finally, data on experience with advanced hepatic resectional surgery or complex stage IV colorectal cancer patients during training are not evaluated, which could certainly impact the findings.

Despite these limitations, the results are important, and the authors are to be congratulated for bringing this issue into sharper focus. Variability in practice patterns, for many different reasons, is not a new concept, having been documented in other areas of medicine, but these results, in particular, underscore the need for studies that provide clear direction on management, based on high-level clinical evidence. Only with the generation of such data will standardization of care be possible.

\section{REFERENCES}

1. Nathan H, Bridges JF, Cosgrove DP, et al. Treating patients with colon cancer liver metastasis: a nationwide analysis of therapeutic decisionmaking. Ann Surg Oncol. 2012. doi:10.1245/s10434-012-2564-3.

2. In H, Neville BA, Lipsitz SR, et al. The role of National Cancer Institute-designated cancer center status: observed variation in surgical care depends on the level of evidence. Ann Surg Oncol. 2012;255(5):890-5. 